\title{
A narrative review of long noncoding RNA: insight into neural ischemia/reperfusion mediated by two pathophysiological processes of injury and repair
}

\author{
Liang Cai, Bingdong Zhang \\ Department of Anesthesiology in Cardiovascular Institute, the First Affiliated Hospital of Guangxi Medical University, Nanning, China \\ Contributions: (I) Conception and design: L Cai; (II) Administrative support: L Cai; (III) Provision of study materials or patients: B Zhang; (IV) \\ Collection and assembly of data: L Cai; (V) Data analysis and interpretation: Both authors; (VI) Manuscript writing: Both authors; (VII) Final \\ approval of manuscript: Both authors. \\ Correspondence to: Bingdong Zhang. Department of Anesthesiology in Cardiovascular Institute, the First Affiliated Hospital of Guangxi Medical \\ University, No. 6 Shuangyong Road, Qingxiu District, Nanning 530021, China. Email: zbd2019@126.com.
}

Background and Objective: A thorough understanding of the role of long noncoding RNAs (lncRNAs)
in cerebral ischemia/reperfusion injury (IRI) is conducive to a comprehensive understanding of the molecular
regulatory network of IRI. Such an understanding could be of great significance for finding new biomarkers
and therapeutic targets of IRI. Such findings could protect important tissues and organs and improve the
clinical prognosis of patients. Methods: We conducted a literature search for published manuscripts on neural ischemia/reperfusion up to October 2021 in the PubMed, Web of Science, Cochrane Library, and EMBASE databases.

Key Content and Findings: LncRNAs are a group of regulatory sequences that play a role at the transcriptional, posttranscriptional and epigenetic levels. LncRNAs are highly expressed in the central nervous system and play an important regulatory role in the development of the central nervous system and diseases. The mechanism of IRI is complex, and pathological injury processes, such as apoptosis, oxidative stress injury, neuroinflammation, excitatory amino acid toxicity, autophagy and impaired blood-brain barrier function, are mutually intertwined or promoted. These processes can aggravate the secondary injury of brain tissue after ischemia-reperfusion. Moreover, cerebral IRI can induce a large number of changes to the expression of lncRNAs in the brain, suggesting that lncRNAs are related to the complicated pathological process of cerebral IRI.

Conclusions: In this narrative review, the roles of lncRNAs in cerebral IRI, apoptosis, anti-apoptosis, nerve regeneration, and repair after injury are reviewed. Additionally, possible future research directions for lncRNAs in ischemic stroke injury and repair are proposed.

Keywords: Long-chain noncoding RNA; cerebral ischemia/reperfusion injury; oxidative stress

Submitted Dec 24, 2021. Accepted for publication Feb 16, 2022.

doi: $10.21037 /$ atm-22-268

View this article at: https://dx.doi.org/10.21037/atm-22-268

\section{Introduction}

Ischemia reperfusion (IR) is a common pathological process that participates in a wide range of diseases, such as myocardial infarction, acute ischemic stroke, acute kidney injury, trauma, and circulatory failure. Restoring the blood supply to ischemic tissue in a timely manner is the best treatment strategy to improve the patient's condition. However, the recovery of the blood supply (1) also brings new damage to ischemic tissues and organs, namely, ischemia/reperfusion injury (IRI). Long noncoding RNAs (lncRNAs) has become a research hotspot in recent years. 
Table 1 The search strategy summary

\begin{tabular}{l} 
Items \\
\hline Date of Search (specified to date, month and year) \\
Databases and other sources searched \\
Search terms used (including MeSH and free text search terms and filters) \\
(Note: please use an independent supplement table to present detailed \\
search strategy of one database as an example) \\
Timeframe \\
Inclusion and exclusion criteria (study type, language restrictions etc.)
\end{tabular}

Selection process (who conducted the selection, whether it was conducted independently, how consensus was obtained, etc.)

\section{Specification}

Up to October 2021

PubMed, Web of Science, Cochrane Library, and EMBASE databases

"neural ischemia/reperfusion", "injury", "repair", and "long noncoding RNA"

Up to October 2021

Qualitative and quantitative data were extracted by interpreting each paper in cycles to avoid missing potentially valuable data

Qualitative and quantitative data were extracted by interpreting each paper in cycles to avoid missing potentially valuable data

No

Any additional considerations, if applicable

Many studies have proven that lncRNAs are involved in the pathological process of IRI in many important organs, such as the heart, brain, liver, kidney and vascular endothelium (2). Some lncRNAs have been proven to be biomarkers of IRI in brain tissue. In the future, lncRNAs may be a potential therapeutic target for preventing stroke IRI (3). Some lncRNAs have been proved to be biomarkers of IR. In the future, it may become a potential therapeutic target for the prevention of IR. Comprehensively exploring the biological function and molecular mechanism of lncRNAs is of great significance for us to study how to protect IRI of tissues and organs. It is of great significance for us to study how to protect tissues and organs from IRI by comprehensively exploring the biological functions and molecular mechanisms of lncRNAs. We present the following article in accordance with the Narrative Review reporting checklist (available at https://atm.amegroups.com/article/ view/10.21037/atm-22-268/rc).

\section{Methods}

We conducted a literature search for published manuscripts on neural ischemia/reperfusion up to October 2021 in the PubMed, Web of Science, Cochrane Library, and EMBASE databases. We used the following search words and terms: "neural ischemia/reperfusion", "injury", "repair", and "long noncoding RNA". Qualitative and quantitative data were extracted by interpreting each paper in cycles to avoid missing potentially valuable data (Table 1).

\section{Discussion \\ LncRNA}

LncRNAs exist in the nucleus or cytoplasm of organisms. They do not encode proteins and are the transcription products of genes encoding very short polypeptides. At present, there is no unified view about the sources of lncRNAs (4). The recognized sources mainly include mutations of protein-coding genes, chromatin recombination, reverse transcription of noncoding genes, duplication of internal segments of noncoding RNA and insertion of transposable elements (5). RNA acts as a medium to regulate the internal conversion of pathways and signals. Four core nucleotides and a variety of chemically modified nucleotides regulate and stabilize the structure of RNA. LncRNAs are usually long, and they form poly A tails and promoter structures by splicing. Then, they form thermodynamically stable secondary or higher structures to perform their function after folding. Compared with coding RNAs, IncRNAs are more susceptible to species evolution, and their sequences are less conserved. More and more lncRNAs can be found in the genomes of organisms, and there are relatively conserved short sequences in their molecules, which are closely related to the important physiological and biochemical functions of expression (6). Therefore, lncRNAs have the characteristics of a specific secondary spatial structure, low sequence conservation but relatively conservative internal short sequences, developmental specificity, dynamic expression 
during differentiation, and various shearing forms. RNA not only plays an auxiliary role as the intermediate carrier of genetic information but also plays a more important role in various regulatory functions (7). Similarly, lncRNAs have complex biological functions at the genetic and transcription levels. In recent years, it has been found that the functions of lncRNAs at the molecular level mainly include three aspects. First, chromatin modifying proteins are collected to regulate gene expression (8). It has been found that the inactivation-specific transcript of the $\mathrm{X}$ chromosome transcribed from the $\mathrm{X}$ chromosome can recruit multicomb suppression complex 2. This can inhibit gene transcription on the $\mathrm{X}$ chromosome, inactivate the genetic traits of one $\mathrm{X}$ chromosome in mammalian females, and achieve the goal of maintaining the same gene phenotype of male and female organisms (9). In addition, lncRNAs related to chromosome modifications other than the $\mathrm{X}$ chromosome are being continuously identified, and such lncRNAs include the Kcnq1 antisense transcript and Igf2 antisense transcript. Second, when combining with DNA or some RNA through base complementation lncRNAs play a regulatory role at the transcriptional and posttranscriptional levels by modifying the promoter structure, changing the cleavage site and covering the binding site. For example, an Alu sequence lncRNA regulates the transcription process by combining with RNA polymerase II to achieve the goal of gene suppression (10). Third, lncRNAs directly affect the function of proteins by changing their location in the cytoplasm, combining with specific proteins and forming nucleic acid-protein complexes. For example, lncRNA ADORA2A was found to exerts tumor-suppressive roles via binding the RNA stable protein $\mathrm{Hu}$ antigen $\mathrm{R}$ and repressing Fascin ActinBundling Protein 1 (11).

\section{LncRNA and IR}

The brain is the most sensitive organ to the ischemic response. Cerebral ischemia will cause irreversible damage to brain tissue in less than $20 \mathrm{~min}$. IR-induced brain microvascular endothelial cell injury is the initial stage of blood-brain barrier injury, which leads to poor prognosis in patients with ischemic stroke (12). Studies have proven that autophagy has a protective effect on ischemic injury in brain microvascular endothelial cells. Li et al. (13) found that the level of metastasis-associated lung adenocarcinoma transcript 1 (MALAT1) in brain microvascular endothelial cells was significantly increased in an IR mouse model. Furthermore, the expression of ULK2 was downregulated by inhibiting the level of miR$26 \mathrm{~b}$, which promoted autophagy and cell survival of brain microvascular endothelial cells. Wang et al. (14) found that MALAT1 expression increased PI3K activity and AKT phosphorylation but decreased Caspase-3 activity in an IR model of brain microvascular endothelial cells. It also inhibited the apoptosis of brain microvascular endothelial cells. Zhang et al. (15) found that the level of MALAT1 was significantly increased after IR in mouse models of both brain microvascular endothelial cell IR and brain IR. Studies have shown that MALAT1 can inhibit apoptosis by inhibiting the levels of the proapoptotic factor BIM and the proinflammatory factors MCP-1, IL- 6 and endothelial cell selectin, thus protecting against IRI in cerebral apoplexy. Extensive studies have shown that IRI not only causes byain microvascular endothelial damage but also triggers a series of neural events, such as hypoxia, oxidative stress, neurotoxicity, inflammatory reaction and edema formation, leading to the death of neurons in ischemic areas (16). Wu et al. (17) detected the change in the expression profile of whole genome lncRNAs in a mouse model of brain IRI and found that lncRNA-N1LR may inhibit apoptosis by inhibiting phosphorylation of the 15 th serine residue of the $\mathrm{p} 53$ protein, thus protecting nerve cells. Li et al. (18) also screened lncRNAs after IRI in mouse hippocampal neurons with high throughput and found that IncRNA-Tnxa-psi promoted the survival of neurons by inhibiting apoptosis.

\section{Mechanisms of noncoding RNAs in the IR process}

\section{Cell damage and death}

Reperfusion injury after cerebral ischemia refers to brain cell damage in the ischemic area that continues to worsen after blood flow is restored. Cerebral ischemia is the first disabling factor, and reperfusion injury after cerebral ischemia is harmful. Therefore, effective control of reperfusion injury is of great benefit to patients with cerebral ischemia. Thai et al. (19) found that the endotoxins in damaged cells were significantly increased and the cell survival rate was significantly decreased after the knockout of a lncRNA named SCAL1, which indicates that SCAL1 plays an important role in cell survival. The NF- $\kappa$ B pathway is a cellular and molecular pathway closely related to IRI.

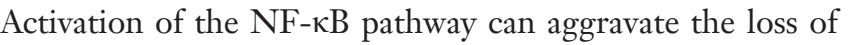
integrity of the blood-brain barrier when injury occurs (20). 
After the blood barrier is destroyed, many inflammatory factors further aggravate cell damage through blood vessels with increased permeability. Additionally, the expression of specific lncRNAs in vascular endothelial cells is increased, which regulates cell damage. The extra-domain A (EDA) is an extra domain of alternative splicing exons encoding type III repeats, and it is closely related to the tissue injury response. Studies have shown that some lncRNAs are related to EDA production $(21,22)$. Apoptosis is an active process under the action of a series of gene activation, expression and regulation. After cerebral ischemia, injured and necrotic cells are mainly located in the ischemic central area, while apoptotic cells are located in the peripheral areas. Apoptosis of brain cells is not necrosis. Many neurons in the ischemic penumbra or infarct area have the potential to recover after a period of ischemia (23). Inhibition of apoptosis is of great help to the prognosis of stroke patients. It has been reported that Zusanli electroacupuncture can inhibit apoptosis and help to improve cerebral ischemia (24). P53 is a tumor suppressor gene, and its encoded protein is a transcription factor that controls the start of the cell cycle. When the cell is damaged and cannot be repaired, the p53 protein participates in the start-up process and causes damaged cells to die via apoptosis (25). The expression of p53 is rapidly increased in ischemic stroke. Recent studies have shown that IncRNA-ROR negatively regulates the expression of $\mathrm{p} 53$ by inhibiting the binding of hnRNPI to 533 mRNA, which is induced by stress. These findings suggest that lncRNA-ROR indirectly regulates apoptosis after cerebral ischemia. Zhou et al. (26) that lincRNA-p21 can directly bind to the MDM2 protein and inhibit the binding of P53 and MDM2, thus affecting the function of p53 (Figure 1).

\section{Excitotoxicity}

Excitotoxicity is one of the important mechanisms of ischemic brain injury. Under conditions of anoxia and glucose deficiency, the energy metabolism of cells is obstructed. Such obstructions can lead to a decrease in ATP-dependent sodium and potassium pump activity, a significant increase in extracellular $\mathrm{K}^{+}$, membrane depolarization, a large release of excitatory amino acids, excessive excitation of postsynaptic neurons, the activation of ionic glutamate receptors on cell membranes, the promotion of extracellular $\mathrm{Ca}^{2+}$ influx, the aggravation of intracellular calcium overload, and a series of events, such as cell swelling, necrosis and apoptosis (26). Ionic glutamate receptors can be divided into NMDA receptors and AMPA receptors (27). The AMPA family includes GluR1-44 subunits, among which the GluR2 subunit can regulate the permeability of the AMDA receptor to $\mathrm{Ca}^{2+}$. However, REST can facilitate gene expression by upregulating the activity of the gene promoter of the GluR EAAT2, which affects $\mathrm{Ca}^{2+}$ influx after excitotoxicity brain injury $(28,29)$. Recently, it has been found that under the induction of stroke, more lncRNA binds to the REST coinhibitors Sin $3 \mathrm{~A}$ and coREST and regulates REST-mediated gene silencing at the epigenetic level (30).

\section{Oxidative}

Nerve cells and endothelial cells produce a large amount of reactive oxygen species (ROS) during mitochondrial respiratory chain injury and reperfusion after ischemia. ROS can damage proteins and DNA and transmit cell necrosis and apoptosis signals (31). ROS can directly activate the mitochondrial permeability transition pore (MPTP) on the mitochondrial membrane or induce p53 to bind to cyclophilin D to open the MPTP. This binding can lead to mitochondrial swelling and further blockage of the mitochondrial respiratory chain and ultimately promotes cell necrosis in the ischemic core area (32). In addition, ROS can activate p38MAPK and mediate the apoptosis of ischemic penumbra cells through the caspase pathway. The pathological process of ischemic stroke can activate two antioxidant pathways: the superoxide dismutase pathway and the Nrf2/ARE pathway $(33,34)$. Signal transduction and activator of transcription 3 (STAT3) is inactivated during reperfusion injury, which leads to a decrease in SOD2 gene expression regulated by STAT3. Bioinformatics methods predict that 120 lncRNAs contain STAT3 binding sites, which suggests that lncRNAs may regulate the expression of SOD genes through STAT3 (35). Under physiological conditions, Keap1 binds to Nrf2, inhibiting its biological activity (36). Under oxidative stress, ROS promote its separation. Then, free Nrf2 binds to the ARE, which induces the expression of antioxidant proteins. Recent studies have shown that SCAL1, a newly discovered lncRNA, increases SCAL1 expression in the endothelial cells of patients with lung cancer caused by smoking. SCAL1 is the downstream molecule of Nrf2. SCAL1 knockout leads to an increase in endotoxins and a decrease in the cell survival rate, which means that SCAL1 plays an important role in the Nrf2/ARE antioxidant pathway (19) (Figure 2). 


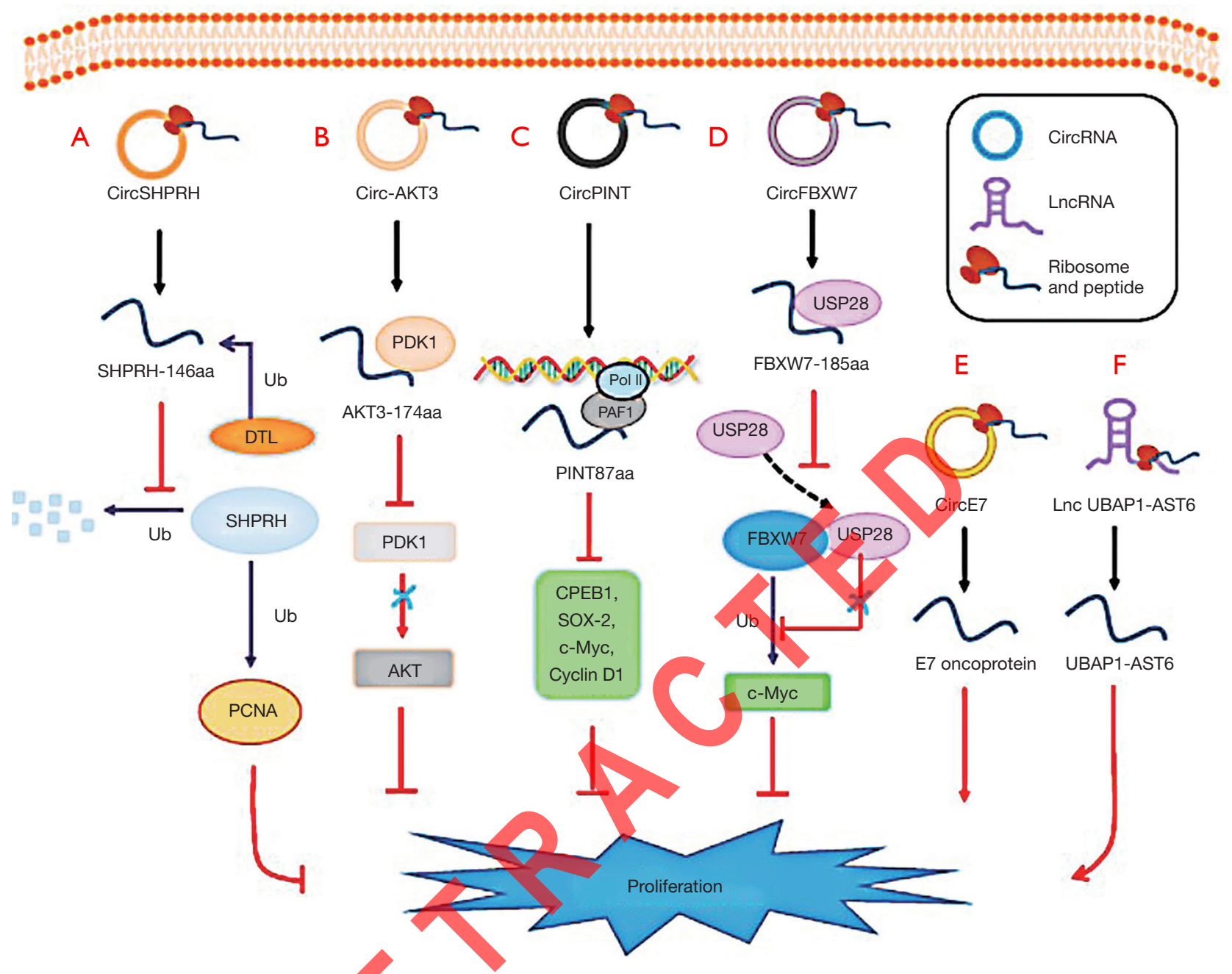

Figure 1 Mechanism of lncRNAs in ischemia-reperfusion injury. SHPRH, SNF2 histone linker PHD RING helicase; AKT3, AKT serine/ threonine kinase 3; FBXW7, F-box and WD repeat domain containing 7; PDK1, pyruvate dehydrogenase kinase 1; DTL, denticleless E3 ubiquitin protein ligase homolog; PCNA, proliferating cell nuclear antigen; USP28, ubiquitin specific peptidase 28.

\section{Inflammatory}

Ischemic stroke rapidly leads to early local inflammatory reactions, including microglial activation and cytokine secretion. With the extension of time, the infiltration of consanguineous macrophages and lymphocytes is also involved in the pathological process of ischemic stroke (37). In the brain, TLR is mainly expressed on the surface of microglia, astrocytes and oligodendrocytes and plays a regulatory role in the activation of the inflammatory response and the secretion of cytokines after cerebral ischemia. Activation of TLR2 after cerebral IRI can cause microglia to secrete IL-23, IL-17 and other cytokines that can damage neurons (38). The expression level of TLR4 was increased after cerebral ischemia, and TLR4 could regulate the expression of TNF- $\alpha$, COX-2, iNOS and other cytokines (39). The signal transduction of TLR2 and TLR4 mainly depends on the MyD88-dependent pathway and MyD88-independent pathway, and it eventually leads to the activation of NF- $\mathrm{KB}$ and the production and secretion of cytokines. Chen et al. (40) found that inhibition of lncRNAAK139328 can significantly decrease the level of Akt in liver IRI. Additionally, Akt binds to $\mathrm{NF}-\kappa \mathrm{B}$, which inhibits its nuclear metastasis and reduces the expression of inflammatory factors. Wang et al. (41) also found that ROCK2, which can promote the inflammatory response after cerebral IRI, has a certain relationship with lncRNAs. Covarrubias et al. (42) found that TLR-induced 


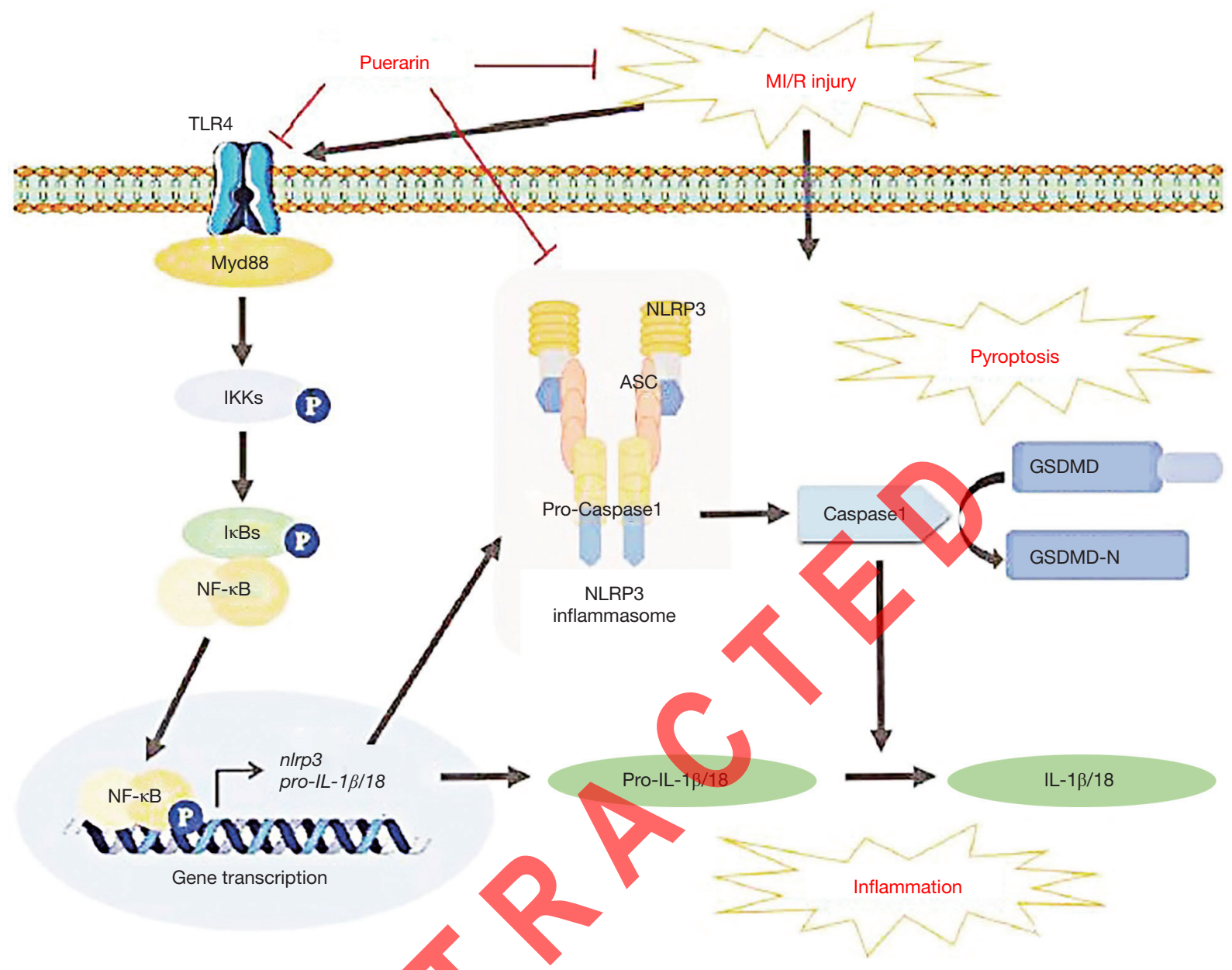

Figure 2 Mechanism of inflammation in ischemia-reperfusion injury. MYD88, MYD88 innate immune signal transduction adaptor; TLR4, toll like receptor 4; NLRP3, NLR family pyrin domain containing 3; GSDMD, gasdermin D.

macrophage lincRNA-Cox2, enhanced the expression of IL-6, and bidirectionally regulated the inflammatory reaction by binding to hnRNPs. All these results indicate that IncRNAs play an important role in the inflammatory response after cerebral IRI.

\section{Destruction of blood barrier}

Cerebral vascular endothelial cells are an important part of the blood-brain barrier (43). Damage to endothelial cells after cerebral ischemia can lead to destruction and dysfunction of the blood-brain barrier, changes in the permeability and integrity of cerebral vessels, vasogenic brain edema, the production of inflammatory factors or the promotion of a large number of inflammatory factors in the ischemic injury area. Such conditions aggravate cell necrosis and apoptosis (44). In cerebrovascular endothelial cells, there is some specific expression of ncRNAs, such as miR-101, miR125a, miR-155, linc00439, Meg3, MALAT1, and TUG1 $(45,46)$. However, little research has been done on their role in injury and protection of the vascular endothelium after stroke (45). Although some miRNAs have been reported to regulate the apoptosis of vascular endothelial cells after stroke and its protective mechanism, the newly discovered role of lncRNAs in vascular endothelial cells after ischemic stroke is still rarely reported (47). This role could provide a new direction for pathological research and the treatment of ischemic stroke in the future.

\section{Role of lncRNA in nerve repair after IR}

For a long time, the central nervous system has not been 
considered to have the function of regeneration after injury. Recently, however, it has been found that there are endogenous neural stem cells (NPCs) in the subventricular zone (SVZ) and subgranular zone (SGZ) of the dentate gyrus of the hippocampus (48). After cerebral ischemia, these neural stem cells begin to differentiate into neurons and glial cells and gradually migrate to ischemic injury areas. This means that nerve repair will become another important treatment measure for ischemic stroke after nerve protection (49). Nerve repair after stroke mainly includes nerve regeneration, blood vessel regeneration, oligodendrocyte regeneration and astrocyte regeneration, which together determine the functional integrity of nerve and blood vessel units after injury (50). Nerve regeneration promotes nerve plasticity and functional recovery. Oligodendrocyte regeneration repairs neuron signal transduction and promotes myelination. Vascular regeneration increases blood flow in the ischemic area and regulates nerve regeneration and glial cell regeneration (51).

\section{Angiogenesis}

Vascular endothelial cells (VECs) are single-layer flat cells lining the lumen of blood vessels, and their activation, proliferation and migration are essential intermediate processes of angiogenesis. Xiong and others $(45,46)$ found that endothelial cells were damaged after cerebral ischemia. They also found that the expression of specific lncRNAs that are highly expressed in vascular endothelial cells, such as linc00493, Meg3, and MALAT1, is increased, which might be related to the injury and repair of endothelial cells (52). Moreover, it was found that the cell cycle of vascular basal endothelial cells was inhibited, the number of basal cells decreased, and cells migrated and germinated to block new blood vessels after MALAT1 was knockedout in mice. These findings suggest that MALAT1 is involved in regulating angiogenesis. Vascular endothelial growth factor (VEGF) can promote the proliferation of vascular endothelial cells $(52,53)$. Its synthesis level is very low in normal tissues of animals and adults, but it is high in embryos and tissues with angiogenesis. VEGF is considered to promote capillary fusion in angiogenesis. There are multiple types of VEGF, including VEGF-A, -B, -C, -D and $-\mathrm{E}$ types. Among these types, VEGF-A can promote the mitosis of endothelial cells and increase the permeability of vascular endothelial cells (54). The VEGF receptors R-1, R-2 and R-3 are distributed on the surface of vascular endothelial cells (55). Huang et al. (56) found that maternal transcript 3 (Meg3) of noncoding RNA can significantly affect the expression of VEGF-A and VEGFR-1, which indicates that $\mathrm{Meg} 3$ is closely related to angiogenesis. Nitric oxide (NO) is an important messenger molecule and cell growth regulator related to blood vessels. Nitric oxide synthase (NOS) is the rate-limiting factor of NO production (57). NOS is usually used to locate the distribution of NO. Endothelial nitric oxide synthase (eNOS) is type of a NOS in endothelial cells. Ling and others have clarified the mechanism of $\mathrm{NO}$ and eNOS in endothelial cells (58). Xu et al. (59) reported that the long noncoding RNA AK094457 plays a role in regulating blood vessels by influencing the production of eNOS. Further research has clarified the mechanism by which the multiprotein complex bound by the eNOS3'UTR can stabilize eNOS mRNA and further interfere with its transcription process to regulate angiogenesis (60).

\section{LncRNA and nerve repair}

Recent research has shown that after the central nervous system is injured by ischemia, the body will trigger a series of reactions to promote the recovery of injured nerves. Jin et al. explained the neuroprotective process of autophagy that occurs in organisms (61). An and others (62) found that after ischemia, some endogenous neural stem cells (NPCs) migrate to the ischemic area and gradually differentiate into neurons and glial cells. Studies have found that more than 30 kinds of lncRNAs are highly expressed in mature neurons. Among these is lncRNAN1, which is involved in regulating gene expression and NPC differentiation. lncRNAN2 can indirectly mediate the occurrence of nerve cells, and the expression of the transcription factor SOX2 can promote NPC differentiation into nerve cells. Some studies have found that lncRNA RMST can bind to the promoter region of the SOX2 target gene and influence the differentiation direction of NPC (63). Recent studies have shown that lncRNAs can also regulate the regeneration and differentiation of neurons by regulating target proteins in the process of nerve regeneration. Glial cells are widely distributed in the central and peripheral nervous systems, and they support and guide the migration of neurons (64). They are divided into astrocytes, oligodendrocytes and microglia, which participate in the repair and regeneration of the nervous system. The main functions of oligodendrocytes are to surround axons, form myelin sheath structures and assist in the transmission of nerve electrical signals. Thus, they maintain and protect 
the normal function of neurons. Studies that analyzed the expression profiles of lncRNAs and mRNAs in injured rat sciatic nerves found 105 lncRNAs that were differentially expressed. They also found that the migration of glial cells was affected, which indicates that lncRNAs could regulate the migration of glial cells (65). In addition, after decreasing the expression level of IncRNA BC089918 in cells, the axons of nerve cells were elongated, which indicates that lncRNAs can inhibit nerve regeneration.

\section{Conclusions}

In summary, lncRNAs are widely involved in various regulatory pathways, such as apoptosis, autophagy, necrosis, and inflammatory reactions, in the process of cerebral ischemia-reperfusion and play an important role. The structure and function of lncRNAs are highly diverse, and lncRNAs play an important role in many human diseases. As a biological marker and therapeutic target, lncRNAs have been widely studied and applied in the field of oncology, but research on lncRNAs in IR is still in its infancy. The specific working mechanism of many lncRNAs is still unknown. In particular, the role of lncRNAs in IRI of lung tissue is unknown, and more research is required to further explore this topic. With the rapid development of science and technology, high-throughput sequencing provides a very convenient method for lncRNA research. There are an increasing number of studies using gene chip technology to discover known and unknown lncRNAs that are differentially expressed in IRI and to further explore their functions. The popularization of this technology is also an inevitable trend for studying lncRNAs in the future. Understanding the functions and mechanisms of these lncRNAs is expected to clarify their complex regulatory mechanisms in IRI.

\section{Acknowledgments}

Funding: None.

\section{Footnote}

Reporting Checklist: Both authors have completed the Narrative Review reporting checklist. Available at https:// atm.amegroups.com/article/view/10.21037/atm-22-268/rc

Conflicts of Interest: Both authors have completed the ICMJE uniform disclosure form (available at https://atm. amegroups.com/article/view/10.21037/atm-22-268/coif). The authors have no conflicts of interest to declare.

Ethical Statement: The authors are accountable for all aspects of the work in ensuring that questions related to the accuracy or integrity of any part of the work are appropriately investigated and resolved.

Open Access Statement: This is an Open Access article distributed in accordance with the Creative Commons Attribution-NonCommercial-NoDerivs 4.0 International License (CC BY-NC-ND 4.0), which permits the noncommercial replication and distribution of the article with the strict proviso that no changes or edits are made and the original work is properly cited (including links to both the formal publication through the relevant DOI and the license). See: https://creativecommons.org/licenses/by-nc-nd/4.0/.

\section{References}

Wang J, Toan S, Zhou H. New insights into the role of mitochondria in cardiac microvascular ischemia/ reperfusion injury. Angiogenesis 2020;23:299-314. Neimark MI. Ischemia-reperfusion syndrome. Khirurgiia (Mosk) 2021;(9):71-6.

3. Lu Y, Han Y, He J, et al. LncRNA FOXD3-AS1 knockdown protects against cerebral ischemia/reperfusion injury via miR-765/BCL2L13 axis. Biomed Pharmacother 2020;132:110778.

4. Chen Y, Li Z, Chen X, et al. Long non-coding RNAs: From disease code to drug role. Acta Pharm Sin B 2021;11:340-54.

5. Senmatsu S, Asada R, Oda A, et al. lncRNA transcription induces meiotic recombination through chromatin remodelling in fission yeast. Commun Biol 2021;4:295.

6. Xu Q, Song Z, Zhu C, et al. Systematic comparison of lncRNAs with protein coding mRNAs in population expression and their response to environmental change. BMC Plant Biol 2017;17:42.

7. Yao X, Yao R, Huang F, et al. LncRNA SNHG12 as a potent autophagy inducer exerts neuroprotective effects against cerebral ischemia/reperfusion injury. Biochem Biophys Res Commun 2019;514:490-6.

8. Zhu J, Fu H, Wu Y, et al. Function of lncRNAs and approaches to lncRNA-protein interactions. Sci China Life Sci 2013;56:876-85.

9. Yang F, Deng X, Ma W, et al. The lncRNA Firre anchors the inactive $\mathrm{X}$ chromosome to the nucleolus by binding 
CTCF and maintains H3K27me3 methylation. Genome Biol 2015;16:52.

10. Chatterjee D, Sanchez AM, Goldgur Y, et al. Transcription of lncRNA prt, clustered prt RNA sites for Mmi1 binding, and RNA polymerase II CTD phospho-sites govern the repression of pho1 gene expression under phosphatereplete conditions in fission yeast. RNA 2016;22:1011-25.

11. $\mathrm{Pu}$ J, Zhang Y, Wang A, et al. ADORA2A-AS1 Restricts Hepatocellular Carcinoma Progression via Binding HuR and Repressing FSCN1/AKT Axis. Front Oncol 2021;11:754835.

12. Liu G, Liang $Y, X u M$, et al. Protective mechanism of Erigeron breviscapus injection on blood-brain barrier injury induced by cerebral ischemia in rats. Sci Rep 2021;11:18451.

13. Li Z, Li J, Tang N. Long noncoding RNA Malat1 is a potent autophagy inducer protecting brain microvascular endothelial cells against oxygen-glucose deprivation/ reoxygenation-induced injury by sponging miR-26b and upregulating ULK2 expression. Neuroscience 2017;354:1-10.

14. Wang G, Wu Y, Zhu Y. Mechanism of MALAT1 preventing apoptosis of vascular endothelial cells induced by oxygen-glucose deficiency and reoxidation. Artif Cells Nanomed Biotechnol 2018;46:798-805.

15. Zhang X, Tang X, Liu K, et al. Long Noncoding RNA Malat1 Regulates Cerebrovascular Pathologies in Ischemic Stroke. J Neurosci 2017;37:1797-806.

16. Sun MS, Jin H, Sun X, et al. Free Radical Damage in Ischemia-Reperfusion Injury: An Obstacle in Acute Ischemic Stroke after Revascularization Therapy. Oxid Med Cell Longev 2018;2018:3804979.

17. Wu Z, Wu P, Zuo X, et al. Erratum to: LncRNAN1LR Enhances Neuroprotection Against Ischemic Stroke Probably by Inhibiting p53 Phosphorylation. Mol Neurobiol 2017;54:7686-8.

18. Li H, Wu Y, Suo G, et al. Profiling neuron-autonomous lncRNA changes upon ischemia/reperfusion injury. Biochem Biophys Res Commun 2018;495:104-9.

19. Thai P, Statt S, Chen CH, et al. Characterization of a novel long noncoding RNA, SCAL1, induced by cigarette smoke and elevated in lung cancer cell lines. Am J Respir Cell Mol Biol 2013;49:204-11.

20. Zhao Y, Li Z, Lu E, et al. Berberine exerts neuroprotective activities against cerebral ischemia/reperfusion injury through up-regulating PPAR- $\gamma$ to suppress NF- $\kappa \mathrm{B}$ mediated pyroptosis. Brain Res Bull 2021;177:22-30.

21. Dhanesha N, Chorawala MR, Jain M, et al. Fn-EDA
(Fibronectin Containing Extra Domain A) in the Plasma, but Not Endothelial Cells, Exacerbates Stroke Outcome by Promoting Thrombo-Inflammation. Stroke 2019;50:1201-9.

22. Oliver-Kozup H, Martin KH, Schwegler-Berry D, et al. The group A streptococcal collagen-like protein-1, Scl1, mediates biofilm formation by targeting the extra domain A-containing variant of cellular fibronectin expressed in wounded tissue. Mol Microbiol 2013;87:672-89.

23. Broughton BR, Reutens DC, Sobey CG. Apoptotic mechanisms after cerebral ischemia. Stroke 2009;40:e331-9.

24. Wang MM, Zhang M, Feng YS, et al. Electroacupuncture Inhibits Neuronal Autophagy and Apoptosis via the PI3K/ AKT Pathway Following Ischemic Stroke. Front Cell Neurosci 2020;14:134

25. Zhao J, Dong X, Chen X, et al. p53 Inhibition Protects against Neuronal Ischemia/Reperfusion Injury by the p53/RRAS40/mTOR Pathway. Oxid Med Cell Longev 2021;2021:4729465.

26. Zhou L, Tian Y, Guo F, et al. LincRNA-p21 knockdown reversed tumor-associated macrophages function by promoting MDM2 to antagonize* p53 activation and alleviate breast cancer development. Cancer Immunol Immunother 2020;69:835-46.

27. Mayor D, Tymianski M. Neurotransmitters in the mediation of cerebral ischemic injury. Neuropharmacology 2018;134:178-88.

28. Pajarillo E, Digman A, Nyarko-Danquah I, et al. Astrocytic transcription factor REST upregulates glutamate transporter EAAT2, protecting dopaminergic neurons from manganese-induced excitotoxicity. J Biol Chem 2021;297:101372.

29. Radchenko EV, Tarakanova AS, Karlov DS, et al. Ligands of the AMPA-subtype glutamate receptors: mechanisms of action and novel chemotypes. Biomed Khim 2021;67:187-200.

30. Mehta SL, Kim T, Vemuganti R. Long Noncoding RNA FosDT Promotes Ischemic Brain Injury by Interacting with REST-Associated Chromatin-Modifying Proteins. J Neurosci 2015;35:16443-9.

31. Juan CA, Pérez de la Lastra JM, Plou FJ, et al. The Chemistry of Reactive Oxygen Species (ROS) Revisited: Outlining Their Role in Biological Macromolecules (DNA, Lipids and Proteins) and Induced Pathologies. Int J Mol Sci 2021;22:4642.

32. Zhang J, Wang X, Vikash V, et al. ROS and ROSMediated Cellular Signaling. Oxid Med Cell Longev 
2016;2016:4350965.

33. Li Y, Shi J, Sun X, et al. Theaflavic acid from black tea protects PC12 cells against ROS-mediated mitochondrial apoptosis induced by OGD/R via activating Nrf2/ARE signaling pathway. J Nat Med 2020;74:238-46.

34. Xiong W, Chen H, Lu J, et al. IL-39 increases ROS production and promotes the phosphorylation of $\mathrm{p} 38$ MAPK in the apoptotic cardiomyocytes. Folia Histochem Cytobiol 2021;59:195-202.

35. Xu G, Zhang $\mathrm{W}$, Wang $\mathrm{Z}$, et al. Matrine regulates $\mathrm{H} 2 \mathrm{O} 2$-induced oxidative stress through long non-coding RNA HOTAIR/miR-106b-5p axis via AKT and STAT3 pathways. Biosci Rep 2020;40:BSR20192560.

36. Hu Y, Xiao T, Wang Q, et al. Effects of Essential Trace Elements and Oxidative Stress on Endemic Arsenism Caused by Coal Burning in PR China. Biol Trace Elem Res 2020;198:25-36.

37. Poittevin M, Deroide N, Azibani F, et al. Glatiramer Acetate administration does not reduce damage after cerebral ischemia in mice. J Neuroimmunol 2013;254:55-62.

38. Sun W, Ding Z, Xu S, et al. Crosstalk between TLR2 and Sphk1 in microglia in the cerebral ischemia/reperfusioninduced inflammatory response. Int J Mol Med 2017;40:1750-8.

39. Li S, Luo L, He Y, et al. Dental pulp stem cell-derived exosomes alleviate cerebral ischaemia-reperfusion injury through suppressing inflammatory response. Cell Prolif 2021;54:e13093.

40. Chen Z, Jia S, Li D, et al. Silencing of long noncoding RNA AK139328 attenuates ischemia/reperfusion injury in mouse livers. PLoS One 2013;8:e80817.

41. Wang J, Fu Z, Wang M, et al. Knockdown of XIST Attenuates Cerebral Ischemia/Reperfusion Injury Through Regulation of miR-362/ROCK2 Axis. Neurochem Res 2021;46:2167-80.

42. Covarrubias S, Robinson EK, Shapleigh B, et al. CRISPR/ Cas-based screening of long non-coding RNAs (lncRNAs) in macrophages with an NF- $\kappa \mathrm{B}$ reporter. J Biol Chem 2017;292:20911-20.

43. Rellick SL, Hu H, Simpkins JW, et al. Evaluation of Bioenergetic Function in Cerebral Vascular Endothelial Cells. J Vis Exp 2016.

44. Yin KJ, Hamblin M, Chen YE. Non-coding RNAs in cerebral endothelial pathophysiology: emerging roles in stroke. Neurochem Int 2014;77:9-16.

45. Ruan W, Li J, Xu Y, et al. MALAT1 Up-Regulator Polydatin Protects Brain Microvascular Integrity and Ameliorates Stroke Through C/EBPß/MALAT1/
CREB/PGC-1 $\alpha /$ PPAR $\gamma$ Pathway. Cell Mol Neurobiol 2019;39:265-86.

46. Xiong W, Qu Y, Chen H, et al. Insight into long noncoding RNA-miRNA-mRNA axes in myocardial ischemia-reperfusion injury: the implications for mechanism and therapy. Epigenomics 2019;11:1733-48.

47. Teng H, Li M, Qian L, et al. Long non-coding RNA SNHG16 inhibits the oxygen-glucose deprivation and reoxygenation-induced apoptosis in human brain microvascular endothelial cells by regulating miR-15a-5p/ bcl-2. Mol Med Rep 2020;22:2685-94.

48. Jinnou H. Regeneration using endogenous neural stem cells following neonatal brain injury. Pediatr Int 2021;63:13-21.

49. Cheng H, Huang Y, Yue H, et al. Electrical Stimulation Promotes Stem Cell Neural Differentiation in Tissue Engineering. Stem Cells Int 2021;2021:6697574.

50. Chen HS, Chen X, Li WT, et al. Targeting RNS/ caveolin-1/MMP signaling cascades to protect against cerebral ischemia-reperfusion injuries: potential application for drug discovery. Acta Pharmacol Sin 2018;39:669-82. Zhang J, Kramer EG, Asp L, et al. Promoting myelin repair and return of function in multiple sclerosis. FEBS Lett 2011;585:3813-20.

52. Li X, Zhang Z, Li A, et al. Propofol attenuates renal ischemia/reperfusion injury by regulating the MALAT1/ miR-126-5p axis. J Gene Med 2021;23:e3349.

53. Cheng C, Xu BL, Sheng JL, et al. LncRNA MALAT1 regulates proliferation and apoptosis of vascular smooth muscle cells by targeting miRNA-124-3p/PPAR $\alpha$ axis. Eur Rev Med Pharmacol Sci 2019;23:9025-32.

54. Melincovici CS, Boşca AB, Şuşman S, et al. Vascular endothelial growth factor (VEGF) - key factor in normal and pathological angiogenesis. Rom J Morphol Embryol 2018;59:455-67.

55. Torres-Vergara P, Rivera R, Escudero C. How Soluble Fms-Like Tyrosine Kinase 1 Could Contribute to BloodBrain Barrier Dysfunction in Preeclampsia? Front Physiol 2021;12:805082.

56. Huang X, Fu C, Liu W, et al. Chemerin-induced angiogenesis and adipogenesis in $3 \mathrm{~T} 3-\mathrm{L} 1$ preadipocytes is mediated by lncRNA Meg3 through regulating Dickkopf-3 by sponging miR-217. Toxicol Appl Pharmacol 2019;385:114815.

57. Fulton DJ, Stepp DW. Protein kinase N2 connects blood flow with NO production in a double AKT. J Clin Invest 2021;131:e154256.

58. Ling Y, Shi J, Ma Q, et al. Vasodilatory Effect of 
Guanxinning Tablet on Rabbit Thoracic Aorta is Modulated by Both Endothelium-Dependent and -Independent Mechanism. Front Pharmacol 2021;12:754527.

59. Xu J, Sun Y, Lu J. Knockdown of Long Noncoding RNA (lncRNA) AK094457 Relieved Angiotensin II Induced Vascular Endothelial Cell Injury. Med Sci Monit 2020;26:e919854.

60. Sun X, Lv H, Zhao P, et al. Commutative regulation between endothelial NO synthase and insulin receptor substrate 2 by microRNAs. J Mol Cell Biol 2019;11:509-20.

61. Jin L, Mo Y, Yue EL, et al. Ibrutinib ameliorates cerebral ischemia/reperfusion injury through autophagy activation and PI3K/Akt/mTOR signaling pathway in diabetic mice.
Bioengineered 2021;12:7432-45.

62. An B, Ma Y, Xu Y, et al. Crocin regulates the proliferation and migration of neural stem cells after cerebral ischemia by activating the Notch1 pathway. Folia Neuropathol 2020;58:201-12.

63. Ng SY, Bogu GK, Soh BS, et al. The long noncoding RNA RMST interacts with SOX2 to regulate neurogenesis. Mol Cell 2013;51:349-59.

64. Ramos AD, Attenello FJ, Lim DA. Uncovering the roles of long noncoding RNAs in neural development and glioma progression. Neurosci Lett 2016;625:70-9.

65. Yu B, Zhou S, Hu W, et al. Altered long noncoding RNA expressions in dorsal root ganglion after rat sciatic nerve injury. Neurosci Lett 2013;534:117-22.

Cite this article as: Cai L, Zhang B. A narrative review of long noncoding RNA: insight into neural ischemia/reperfusion mediated by two pathophysiological processes of injury and repair. Ann Transl Med 2022;10(4):235. doi: 10.21037/atm-22268 